\title{
Carpooling and the Economics of Self-Driving Cars
}

\author{
MICHAEL OSTROVSKY, Stanford University and NBER, USA \\ MICHAEL SCHWARZ, Microsoft, USA
}

\begin{abstract}
We study the interplay between autonomous transportation, carpooling, and road pricing. We discuss how improvements in these technologies, and interactions among them, will affect transportation markets. Our main results show how to achieve socially efficient outcomes in such markets, taking into account the costs of driving, road capacity, and commuter preferences. An important component of the efficient outcome is the socially optimal matching of carpooling riders. Our approach shows how to set road prices and how to share the costs of driving and tolls among carpooling riders in a way that implements the efficient outcome.
\end{abstract}

CCS Concepts: • Theory of computation $\rightarrow$ Algorithmic game theory and mechanism design; Market equilibria; • Applied computing $\rightarrow$ Economics; Transportation.

Additional Key Words and Phrases: congestion pricing, autonomous vehicles, matching, coalition formation

ACM Reference Format:

Michael Ostrovsky and Michael Schwarz. 2019. Carpooling and the Economics of Self-Driving Cars. In The 20th ACM conference on Economics and Computation (EC '19), fune 24-28, 2019, Phoenix, AZ, USA. ACM, New York, NY, USA, 2 pages. https://doi.org/10.1145/3328526.3329625

\section{INTRODUCTION}

The market for transportation will be transformed by three emerging technologies. Autonomous driving technology is the most radical of the coming changes, but there are two other emerging technologies that are less futuristic but potentially no less disruptive: (i) technology for timesensitive tolls that can be charged for each road segment without slowing down cars and (ii) carpooling technology for seamlessly matching cars with multiple passengers. This paper explores the issue of designing efficient transportation markets powered by these three technologies.

Of course, tolls and carpooling are not new. However, the frictions currently associated with road pricing and carpooling are very high. These frictions are likely to decrease substantially in the future because of technological improvements.

In the case of tolls, the first generation of the technology involved human toll collectors charging a payment from each passing car, which had to stop to make the payment. The second (current) generation of toll technology involves costly hardware at the points of toll collection. Both technologies are expensive to install and/or run, are thus limited to only a few major locations, and do not allow toll collection on most road segments. The third (next) generation will reduce the cost of physical toll infrastructure by orders of magnitude, because of advances in GPS, mobile, camera/OCR, and other related technologies. It will become practical to charge tolls systemwide, street intersection by street intersection, allowing regulators to keep roads toll-free during off-peak hours, and to set tolls high enough to keep traffic flowing during rush hour. Technological constraints are not the only potential barrier to the adoption of systemwide tolls: commuters may worry about distributional consequences of such tolls, or about not benefiting from the toll revenue collected by the

Permission to make digital or hard copies of part or all of this work for personal or classroom use is granted without fee provided that copies are not made or distributed for profit or commercial advantage and that copies bear this notice and the full citation on the first page. Copyrights for third-party components of this work must be honored. For all other uses, contact the owner/author(s).

EC '19, fune 24-28, 2019, Phoenix, AZ, USA

(C) 2019 Copyright held by the owner/author(s).

ACM ISBN 978-1-4503-6792-9/19/06.

https://doi.org/10.1145/3328526.3329625 
government. As we explain in the paper, introducing tolls in the presence of frictionless carpooling technology helps substantially alleviate these concerns: the two technologies are complementary.

Carpooling is also an old "technology," with a potentially large upside: putting multiple commuters in a car, instead of having them drive solo, would dramatically cut the number of cars on the roads. Various government initiatives have promoted carpooling for over half a century, yet it still not widely used. Why will the future be different? The answer is, again, technological progress. Perfect carpool partners travel along similar routes at similar times. For many people, there are many compatible carpool partners-however, these partners change day to day, because schedules change. Until recently, it was too hard to find a good carpool partner, at least without advance planning, and too inconvenient to coordinate with him or her. This is starting to change. New mobile phone apps automate the process of finding good carpool matches, and also keep track of user reputation and preferences. The products are still in their infancy, but they demonstrate the potential of technology to substantially lower the frictions associated with carpooling, especially as the technology matures and the network of commuters who use it grows, making carpooling more attractive. But viewing technological progress in carpooling in isolation would present an incomplete picture of its potential.

In the paper, we argue that there are strong interdependencies between improvements in the three technologies we mentioned: autonomous transportation, road pricing, and carpooling. Autonomous transportation makes both road pricing and carpooling more convenient and attractive. Road pricing and carpooling reinforce each other: the former makes the latter more attractive, and vice versa. These observations raise a variety of questions about the efficient design of transportation in light of these technological improvements and interactions among them. How should road prices be set? How should carpooling groups be determined? How much should each of the carpooling passengers pay (e.g., if one passenger is in the car for only a part of the ride, while another passenger is in the car for the whole ride-but the car had to make a small detour to pick up the first passenger)? How should carpooling interact with road pricing (e.g., how should tolls paid by passengers depend on the number of riders in the car)? Our modeling framework is designed to address these questions.

The framework blends together ideas from coalition formation games (riders form coalitions to carpool and share costs) and competitive equilibrium (road prices are set at the level that clears the market). The framework naturally incorporates interactions between the two components: tolls impact which carpooling coalitions get formed; in turn, the carpooling coalition formation process impacts the demand for roads. Our main results show how to achieve socially efficient outcomes in such markets, taking into account the costs of driving, road capacity, and commuter preferences. An important component of the efficient outcome is the socially optimal matching of carpooling riders. Our approach shows how to set road prices and how to share the costs of driving and tolls among carpooling riders in a way that implements the efficient outcome. We find that efficient market design for this setting can be "decomposed" into (i) road pricing by the road authorities (where road segments have positive prices only when they are at capacity, and are free otherwise) and (ii) unconstrained coalition formation in which groups of riders are allowed to arrange whichever carpooling trips they want, and can split the costs of those trips in any way they choose. For concreteness, our main results are established in an economy in which all cars are self-driving. In the paper, we also explain how our model and results can be adapted to a world in which some or all cars are conventional. To the best of our knowledge, this is the first paper that jointly characterizes efficient tolls and efficient carpooling arrangements.

\section{ACKNOWLEDGMENTS}

The first author is grateful to the National Science Foundation for financial support (grant \#1824317).

Extended Abstract. Full paper is available at https://web.stanford.edu/ ost/papers/sdc.pdf. 